\title{
Normalised Root Mean Square and Amplitude of Sidebands of Vibration Response as Tools for Gearbox Diagnosis
}

\author{
P.J. Rzeszucinski ${ }^{1}$, J. K. Sinha ${ }^{1}$, R. Edwards ${ }^{1}$, A. Starr ${ }^{2}$, B. Allen ${ }^{3}$ \\ ${ }^{1}$ School of MACE, The University of Manchester, Manchester, Lancashire, M13 9PL, UK \\ e-mail: pawel.rzeszucinski@postgrad.manchester.ac.uk, jyoti.sinha@manchester.ac.uk, \\ rodger.edwards@manchester.ac.uk \\ ${ }^{2}$ School of Applied Sciences, Cranfield University, Cranfield, Bedfordshire, MK43 0AL, UK \\ e-mail: $\underline{\text { a.starr@cranfield.ac.uk }}$ \\ ${ }^{3}$ QinetiQ Ltd., Farnborough, Hampshire, GU14 0LX, UK \\ e-mail: ballen2@qinetiq.com
}

\section{Corresponding author:}

Dr. Jyoti Kumar Sinha, School of Mechanical, Aerospace and Civil Engineering, The University of Manchester, Pariser Building, Room B3, Manchester, UK, M13 9PL Tel: +44 161306 4639, Fax: +44 161306 4601, email: jyoti.sinha@manchester.ac.uk

\begin{abstract}
Quick assessment of the condition of gearboxes used in helicopters is a safety requirement. One of the most widely used helicopter on-board mounted condition monitoring system these days is the Health and Usage Monitoring System (HUMS). It has been specifically designed to monitor the condition of all safety-critical components operating in the helicopter through calculation of so called Condition Indicators (CIs) - signal processing routines designed to output a single number that represents the condition of the monitored component. Among number of available parameters there is a couple of CIs that over the years of testing have earned a reputation of being the most reliable measures of the gear tooth condition. At the same time however it has been observed that in some cases those techniques do not properly indicate the deteriorating condition with the propagation of a gear tooth fault with the period
\end{abstract}


of operation. Hence three more robust methods have been suggested which are discussed in this paper.

\section{Introduction}

Helicopter drive trains are extremely compact which makes the task of performing proper inflight condition monitoring very difficult. Complex geometry, complicated stress states, difficult to predict crack growth patterns and a very complex load history add to the extreme difficulty in assessing the condition of helicopter components [1].

In a comprehensive analysis of the cause of helicopter accidents [2] NASA investigated that the drive trains to the main and tail rotors were implicated in a $38 \%$ of all accidents for single piston engine helicopter. $27 \%$ of those accidents were due to main or tail rotor gearbox failure, where the direct source of failure was dominated by gear failures. Those types of malfunctions often lead to some very serious consequences including fatalities for example the Eurocopter Super Puma accident that took place in March 2009 where the post-crash preliminary investigation revealed that the accident occurred following a catastrophic failure of the main rotor gearbox which resulted in detachment of main rotor head from the helicopter [3].

Although the science of vibration analysis forms the basis of a number of techniques capable of properly indicating a gear fault, most of them require specialist knowledge, offline analysis of data, advanced signal processing techniques and much user experience of data interpretation in order to determine if a gear fault has developed. Due to the demanding requirements these approaches may be considered inappropriate in a situation when a quick, in-flight, easily interpretable (by a vibration non-specialist for example a helicopter pilot) gear health indication is required.

For this reason on-board mounted helicopter condition monitoring systems, like the Health and Usage Monitoring System (HUMS), tend to rely on the, so called, Condition Indicator (CI) parameters - signal processing routines designed to output a single number that reflect the health of the given drive-train component for example a gear. Systems such as HUMS operate on the basis of calculating a set of such CIs for all monitored components, and comparing them with a bank of preset thresholds [1]. The crossing of the threshold level gives a clear and quick indication of fault progression within the system.

In this paper the robustness of couple of the most popular gear related CIs has been evaluated on an experimental dataset which contained a full history of progressively deteriorating gear condition - from healthy condition until complete tooth detachment. The 
analysis of the results has been followed by the proposal of three new CIs. The first one is based on the Root Mean Square value of a vibration acceleration response normalised by signal's peak value. The second method makes use of information that appears in the spectrum of the vibration signal as the fault progress. The last CI is an improvement of the second proposed CI. The paper discusses the proposed CIs and their results compared to the existing CI parameters.

\section{Vibration based existing CIs}

RMS is based on the Root Mean Square parameter values of a vibration acceleration response signal signal $x(t)$ and can be defined as [4]:

$$
R M S=\sqrt{\frac{1}{N} \sum_{i=1}^{N} x_{i}^{2}}
$$

Because of the way RMS has been designed it does not increase with the isolated peaks in the signal - only a periodic series of high energy events will increase the overall level of vibration, hence increasing the value of RMS. As a result this parameter is not sensitive to incipient tooth failure and starts indicating a fault only after the tooth damage crossed a certain level of severity. The main usage of this parameter is to monitor the overall vibration level and should ideally be used in conjunction with other, load independent parameters [4].

CF is based on the Crest Factor parameter values of a vibration acceleration response signal $x(t)$ and can be defined as the peak value of $x(t)$ divided by the RMS value of the $x(t)[5]:$

$$
C F=\frac{\max |x|}{R M S}
$$

where $\max |x|$ is the maximal absolute value present in the signal, RMS is the Condition Indicator as per Eq. (1). CF was designed to detect early impulses appearing in the signal that are characteristic for an incipient gear fault. As the gear tooth condition deteriorates shortly after a fault development, the impulsive content within the signal increases, boosting the value of the indicators nominator. At the same time the energy within the impulses is not big enough to cause noticeable changes in the values of RMS. This causes the CF values to increase. However, as the damage progress the RMS values start to increase quicker than the maximal absolute amplitude present in the signal which causes the overall $\mathrm{CF}$ value to 
decrease. CF might therefore be useful in indicating the early stages of gear fault development however as the severity of the fault progress, CF values are likely to drop [6].

Kurtosis is a parameter defined as the fourth centralized moment of the signal, normalized by square of the signal's variance. For vibration acceleration response signal $x(t)$ it can be defined as [4]:

$$
\text { Kurtosis }=\frac{N \cdot \sum_{i=1}^{N}\left(x_{i}-\bar{x}\right)^{4}}{\left(\sum_{i=1}^{N}\left(x_{i}-\bar{x}\right)^{2}\right)^{2}}
$$

where $\bar{x}$ is the mean value of $x(t)$. Kurtosis was designed to react according to the shape of the signal's amplitude distribution. If a vibration signal contains events which are impulsive in nature then its overall amplitude distribution function is sharper, leading to higher kurtosis values [4]. However it has been reported that as the gear fault develops from being localised to more widely distributed, the generated vibration acceleration signal becomes less impulsive and transforms into more complex signal containing high energy, more widely distributed components [7]-[9]. This in turn reduces the peakedness of the signal's amplitude distribution which causes the values of Kurtosis to drop.

\section{Experimental data}

The data were collected by the U.S. Navy's Helicopter Transmission Test Facility (HTTF), located at the Naval Air Warfare Center, Patuxent River, Maryland as part of the Helicopter Integrated Diagnostic System (HIDS) programme. The test rig was a SH-60 Sea Hawk helicopter drive train. The objective of the test was to force gear fault propagation from a condition of good working order to a point of near catastrophic failure. In depth investigation revealed that the component of the drive train that had the potential to deliver the objective in the shortest time was the pinion gear located in the Intermediate Gearbox (IGB) as it had one of the highest bending stresses in the entire SH-60 drive train. Figure 1 shows the sketch of the whole test rig drive train with the location of the accelerometers and the location of the Intermediate Gearbox. 


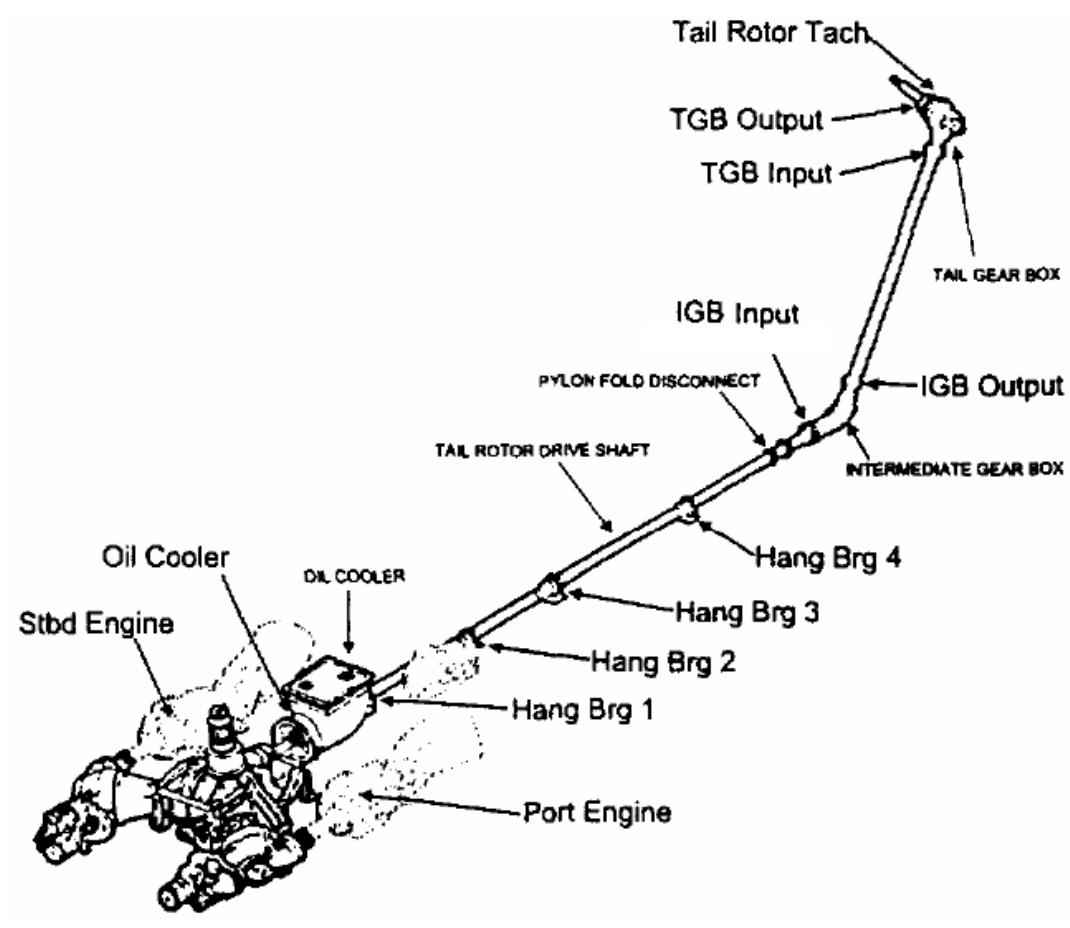

Figure 1. Location of the accelerometers on the test rig drive train [10]

The objective of the test was to force the crack to propagate along a specific path along the length of the root and down into the web of the gear. In order to achieve this, two small notches were seeded on the gear prior to the test. One notch was located near the toe of the tooth, the second near the centre, underneath where the load pattern indicated maximum load for a healthy gear. Both notches were located at the root of the pinion tooth and both were parallel to it.

The test was a constant torque, constant speed arrangement with manual inspections assessing the condition of the gear carried out approximately every 2 hours during the test period. There were a total of 4 inspections carried out after sample 25, 46, 57 and 74. Each inspection consisted of removing the pinion gear from the test rig, removing the lubricant and performing a number of non-destructive tests in order to assess the condition of the gear.

The dataset comprise a total of 85 samples. After recording the last file the test was stopped due to a severe pinion gear crack. Inspection revealed that the fault initiated from the tooth root, extended through the gear web and stopped at a bearing support diameter. A photograph of the gear taken after the test is shown on Figure 2. 


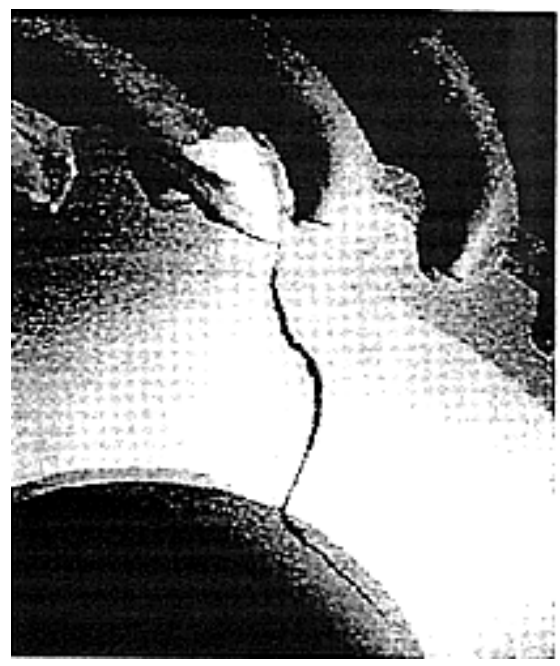

Figure 2. A severe gear crack that led to termination of the test [10]

Data files were recorded every 5 minutes and each sample acceleration response contained 30 seconds of raw vibration acceleration data. The sampling rate of the system was $100 \mathrm{kHz}$. Vibration data used in the analysis was collected by the 'IGB Input' accelerometer (see Figure 1).

\section{Existing CI parameters results}

In order to assess the robustness of CI: RMS, CF and Kurtosis mentioned in Section 2, they are applied on all samples from the dataset under consideration. The results are presented on Figure 3 - Figure 5.

The mean value of RMS stays on constant level until sample 25 when it jumps suddenly. The value does not change until sample 45 where it drops suddenly and continues to decrease until sample 74. After that the value jumps up again and presents both - rising and falling trends just before the gear detachment took place. Based on the above a conclusion can be made that RMS fails to present a meaningful gear fault progress trend (Figure 3).

In the early stages of the fault progress CF reacts quickly to the single impulses present in the signal (sample 25) and continues to follow an increasing trend, however after sample 70 the $\mathrm{CF}$ values drop. As the fault develops it starts to interact with the adjacent teeth increasing the overall vibration level hence raising the denominator in the CF formula. This in turn leads to a drop in the CI values towards the end of the test (Figure 4). Such indication could lead to some inaccurate conclusions being drawn about the condition of the component being monitored.

Kurtosis presents an overall constant, slowly rising trend however its values start to increase late into the test (at sample 46). In addition to that the trend is rising only until 
sample 75 when a noticeable drop in the values can be observed. This event is followed by slight increase in the values. Only the last three samples in the dataset are indicated by Kurtosis as a major fault which results in a considerable increase in the values (Figure 5). In general the behaviour of Kurtosis makes it an unsuitable tool for early gear fault detection due to its late indication of fault development.

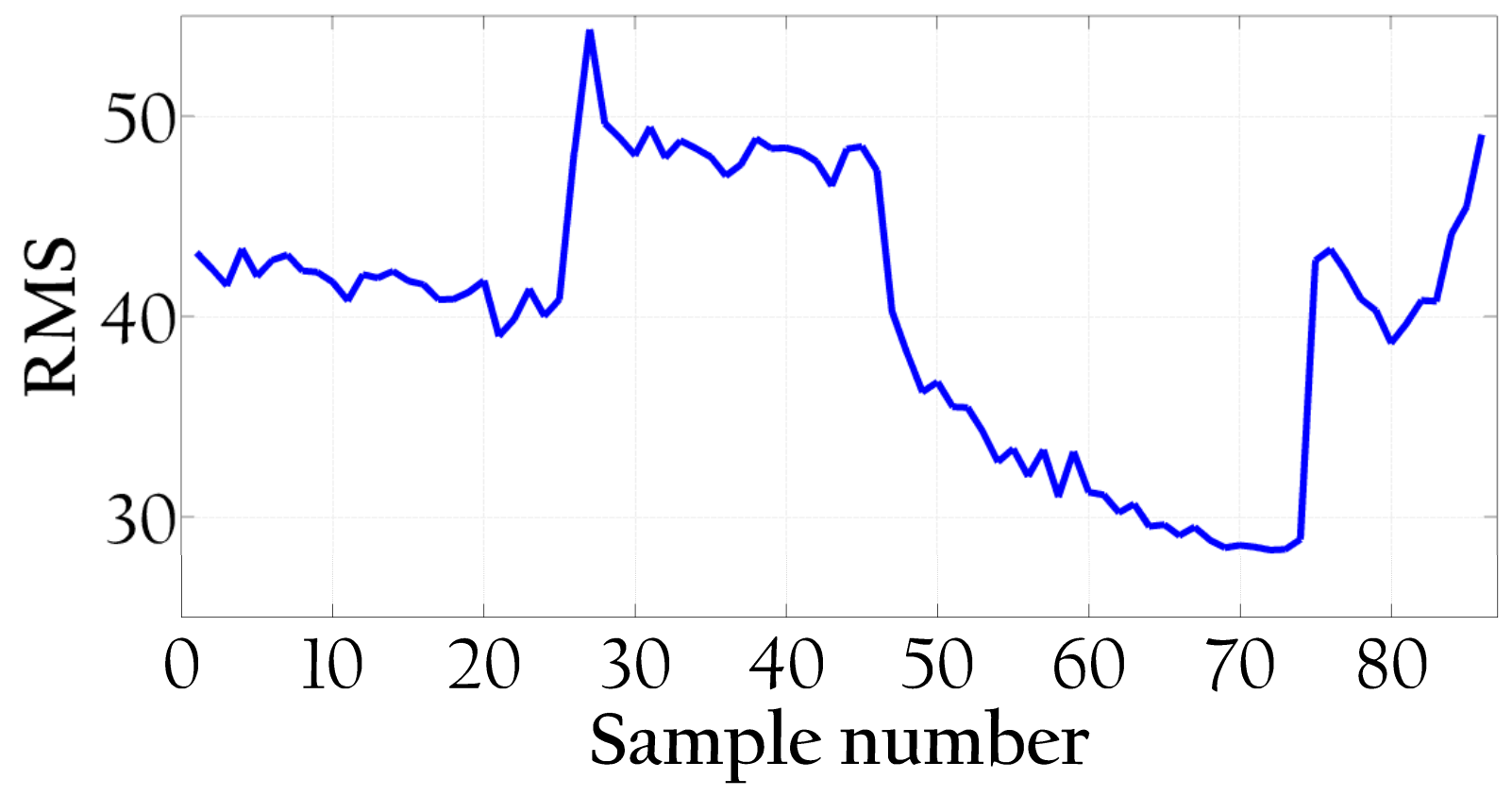

Figure 3. Values of RMS with fault progress

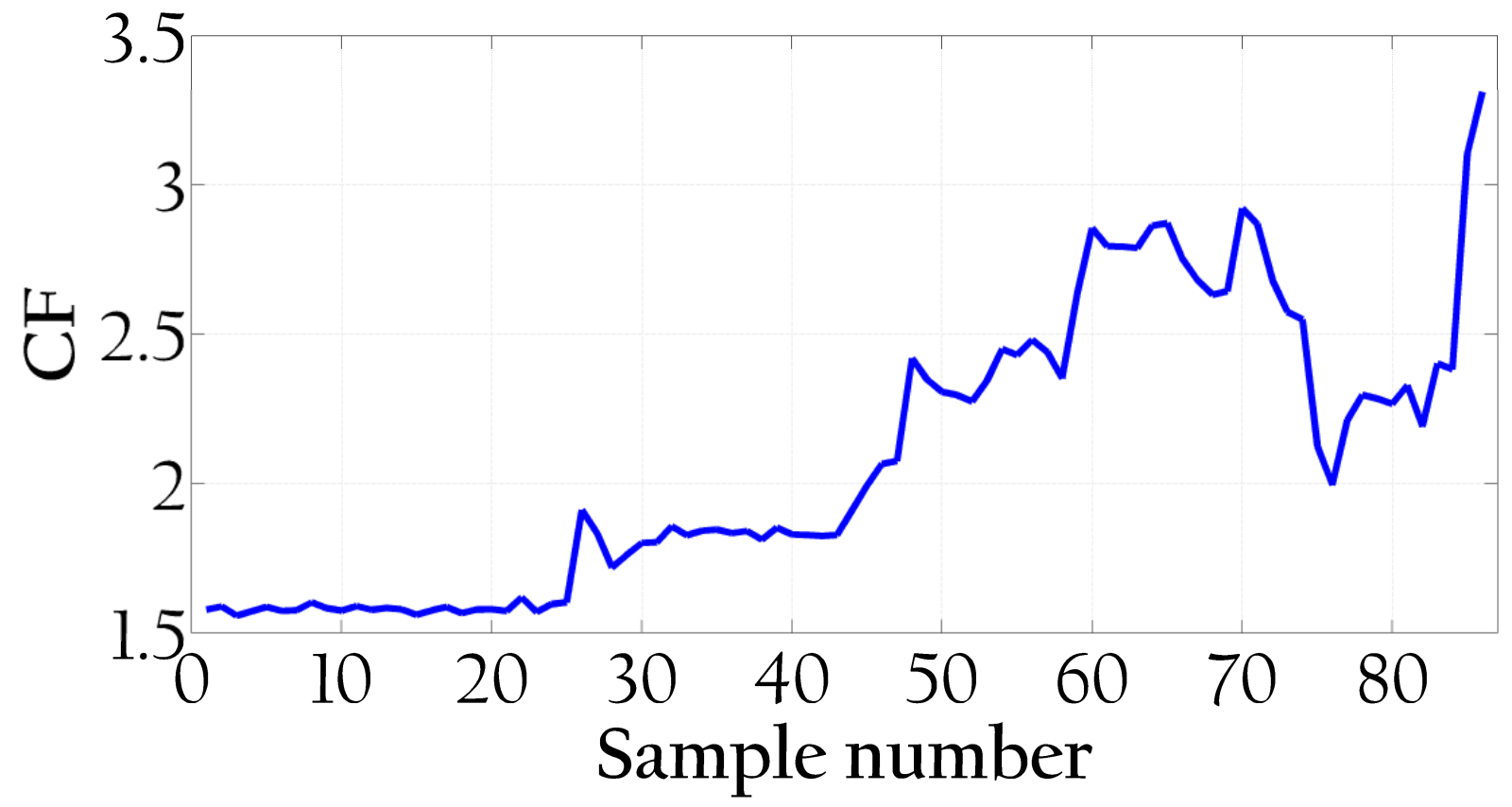

Figure 4. Values of CF with fault progress 


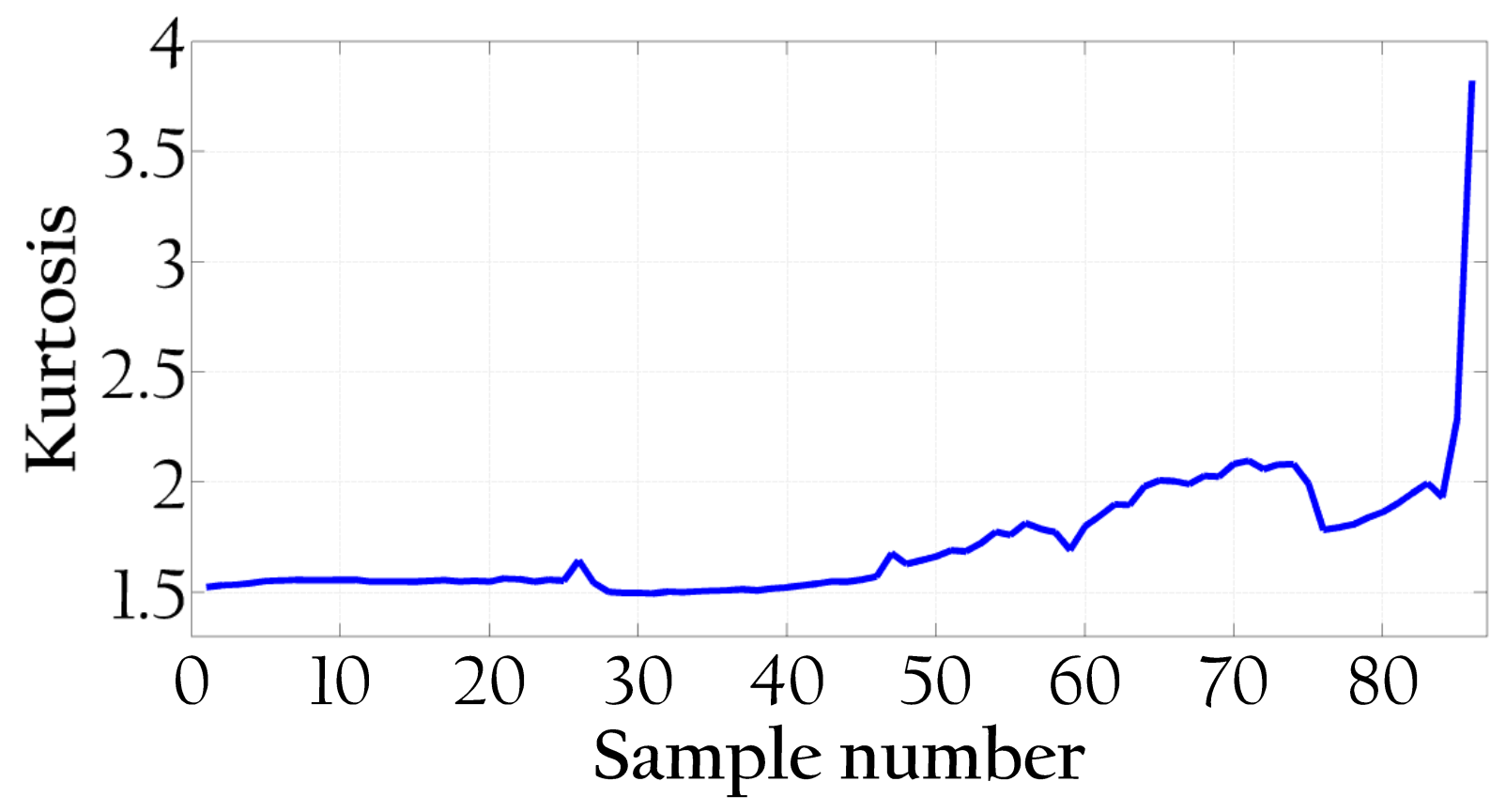

Figure 5. Values of Kurtosis with fault progress

Based on the outcomes of the comparison it is clear that RMS has not been capable of indicating a progressing gear failure in a form of constantly rising values. Kurtosis has been able to increase its values as a function of deterioration of the gear condition, but the indication has begun very late into the test, when the gear fault has reached advanced stages. $\mathrm{CF}$ has shown the best initial indication. The fault has been detected at its early stages, which has been followed by the increase in CF values until sample 70. After that point however the values of the parameter have dropped which has been caused by an increase in the complexity of the generated vibration signal due to fault spreading from being localised to affecting higher number of teeth. The comparison of results generated by the existing CIs clearly showed that in some situations they are not capable of meaningfully and/or continuously indicating a gear fault, therefore they are not suitable for the purpose of performing a robust gear condition monitoring task in helicopters. For that reason three new Condition Indicators are proposed.

\section{Proposed method 1 - nRMS}

The new CI is called "normalised RMS" (nRMS) and it operates on the basis of calculating Root Mean Square of a vibration acceleration signal with amplitude normalised by signal's peak value. nRMS derived for vibration acceleration signal $x(t)$ can be mathematically described as: 


$$
n R M S=1-\frac{R M S(x(t))}{|\max (x(t))|}
$$

where RMS is CI as per Eq. (1), $|\max (x(t))|$ is the maximal absolute value present in the vibration acceleration signal $x(t)$.

The advent of a gear fault leads to generation of high energy events within the vibration signal. As the gear fault develops from being localised to being more widely distributed, the generated signal becomes less impulsive and transforms into a more complex signal containing high energy, more widely distributed components. This in turn leads to increase in the value of the RMS values and decrease isolation of peaks within the vibration acceleration signal. At the same time since the normalisation of the vibration signal always limits the maximal absolute value of the signal to \pm 1 then, as the fault-related events start to appear, the amplitude of the central part of the signal's distribution will decrease.

\subsection{Analysis and observations}

In order to better understand the behaviour of nRMS a set of chronologically arranged vibration acceleration signals are illustrated on Figure 6 - Figure 11. For easier comparison the amplitude of the vibration signals is normalised to \pm 1 .

Figure 6 does not present any clear signatures of frequencies other that the GMF. Also the amplitude of the vibration acceleration signal shows similar value for the entire shaft revolution. Signal derived from sample 24 starts to show signatures of incipient gear fault through an increase in the signal amplitude in the region around 0.009s (Figure 7). Sample 45 supports the findings from previous case as the difference in the amplitude between signal before and after $0.009 \mathrm{~s}$ becomes more evident (Figure 8). In addition to the increase in the amplitude it can be observed that the frequency content in the signal around $0.009 \mathrm{~s}$ starts to become more complex which manifests itself through the presence of additional sharp edges within the waveform. Figure 9 shows the effect of further deterioration of gear fault. The additional high frequency components start to appear in every waveform present in the signal. At the same time the amplitude difference between the faulty and the healthy parts of the vibration acceleration signal becomes more prominent. In contrast to the previous case, signal of sample 75 shows a decrease in the amplitude difference between the faulty and the healthy parts of the signal (Figure 10). This might be due to the fact that this sample was recorded after the last manual inspection which might have temporarily decreased the influence of the faulty gear on the whole gearbox. Signal derived from sample 85, recorded moments before 
the test was terminated, clearly shows the extent of damage that the gear suffered when compared to Figure 6. The shape of the waveform in the whole length of the signal is very jagged which indicates a high amount of complex and widely distributed events appearing during such severe gear failure as a crack.

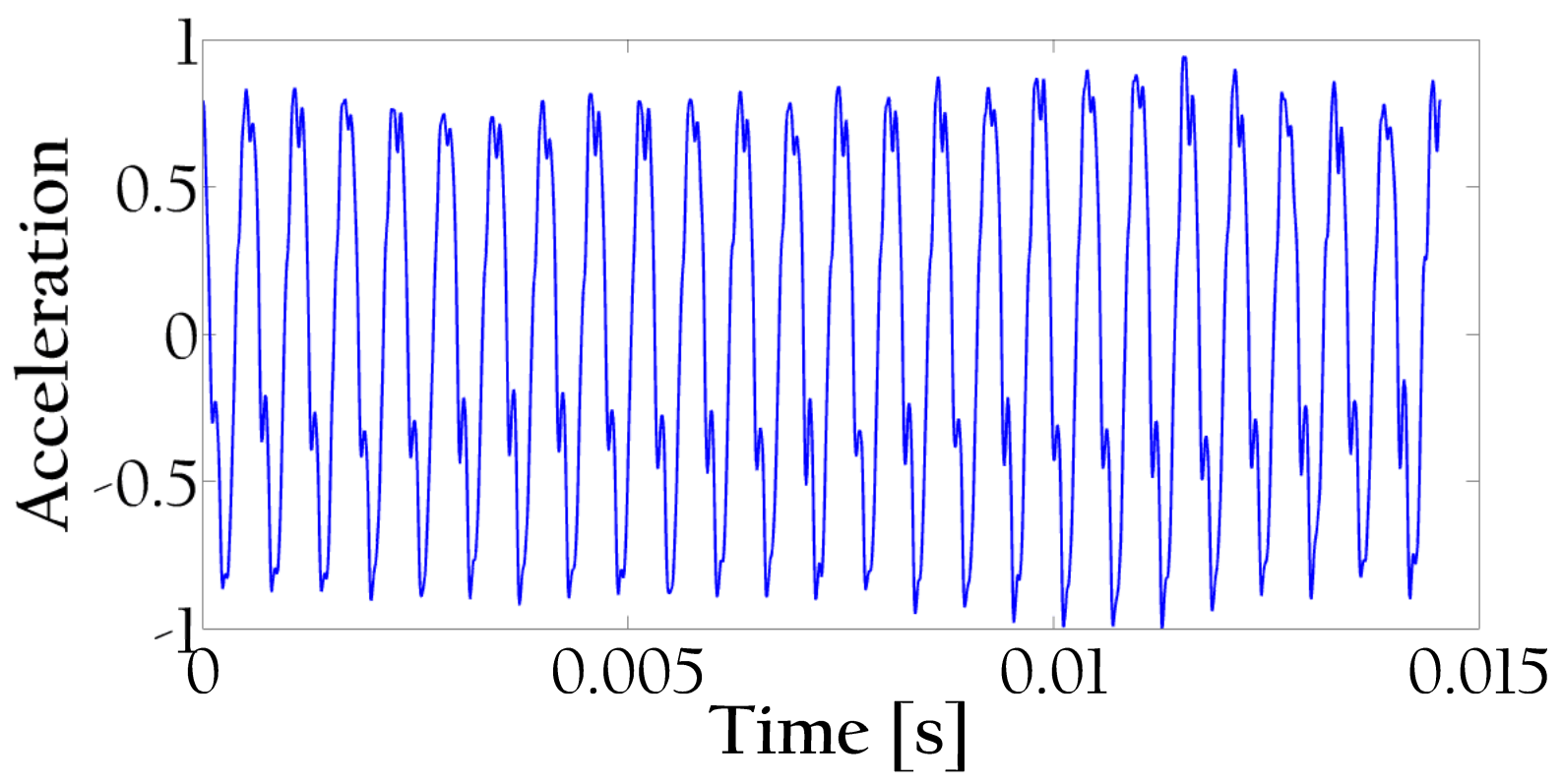

Figure 6. Normalised vibration acceleration signal derived from sample 1

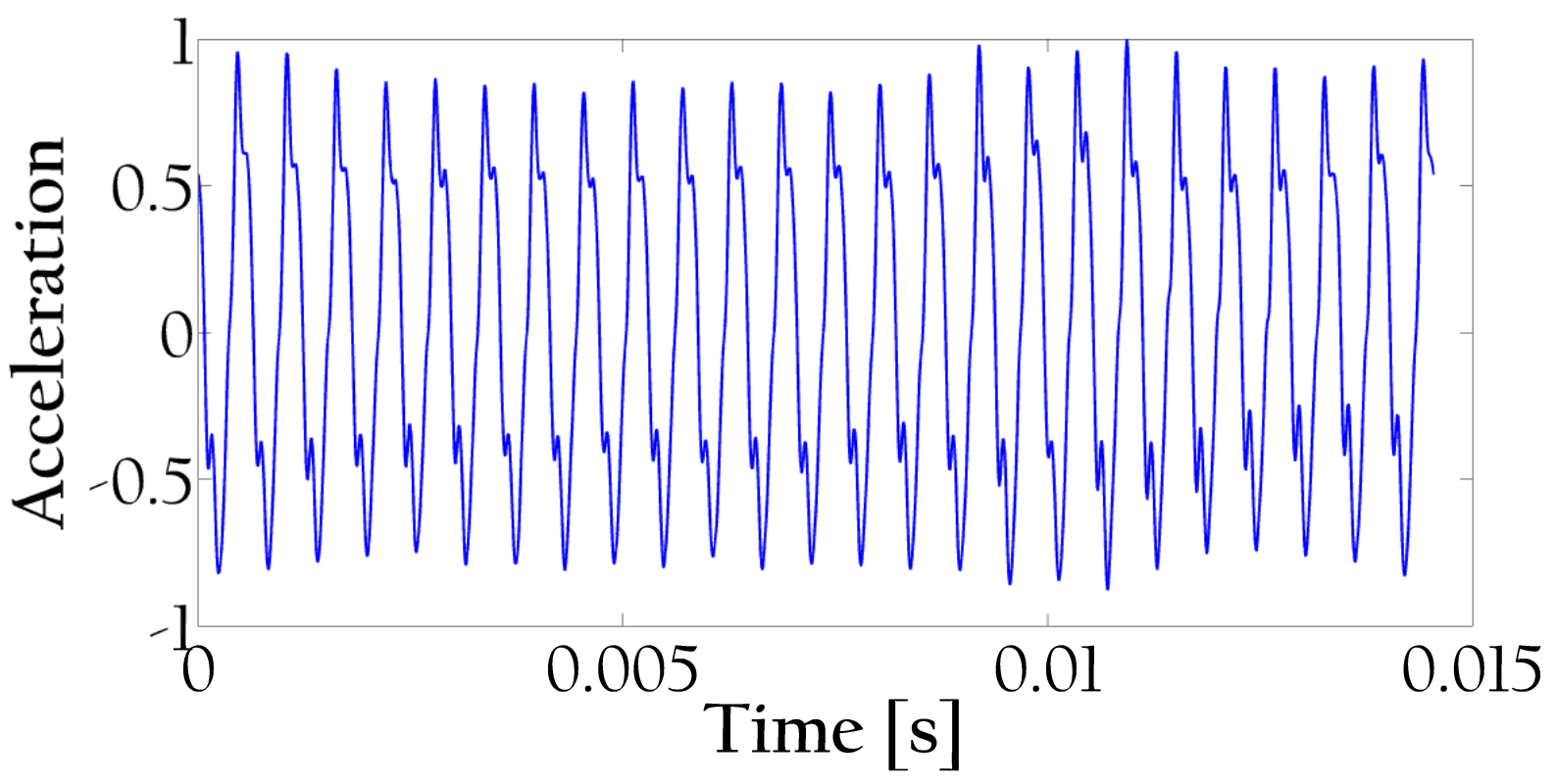

Figure 7. Normalised vibration acceleration signal derived from sample 24 


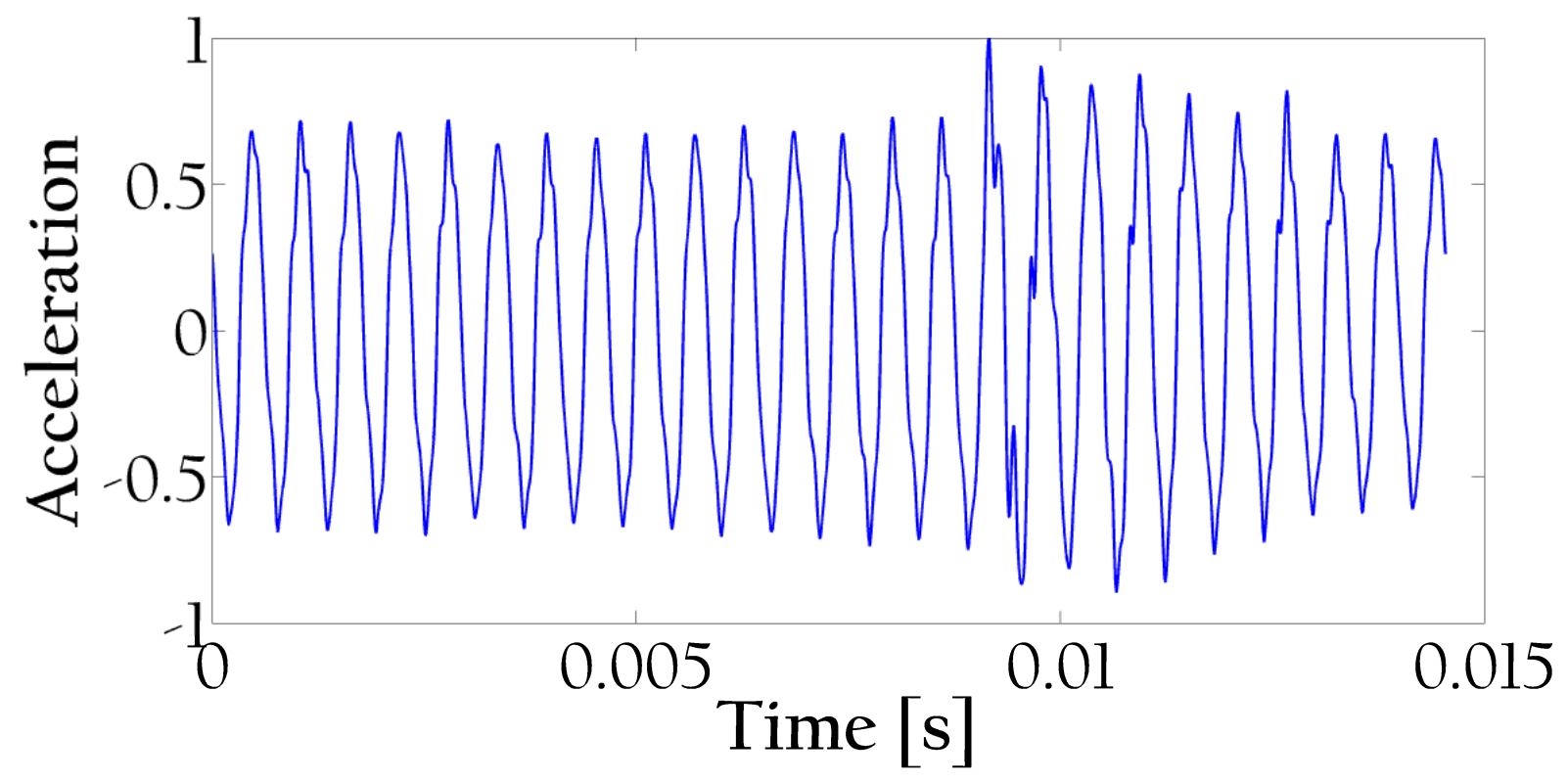

Figure 8. Normalised vibration acceleration signal derived from sample 45

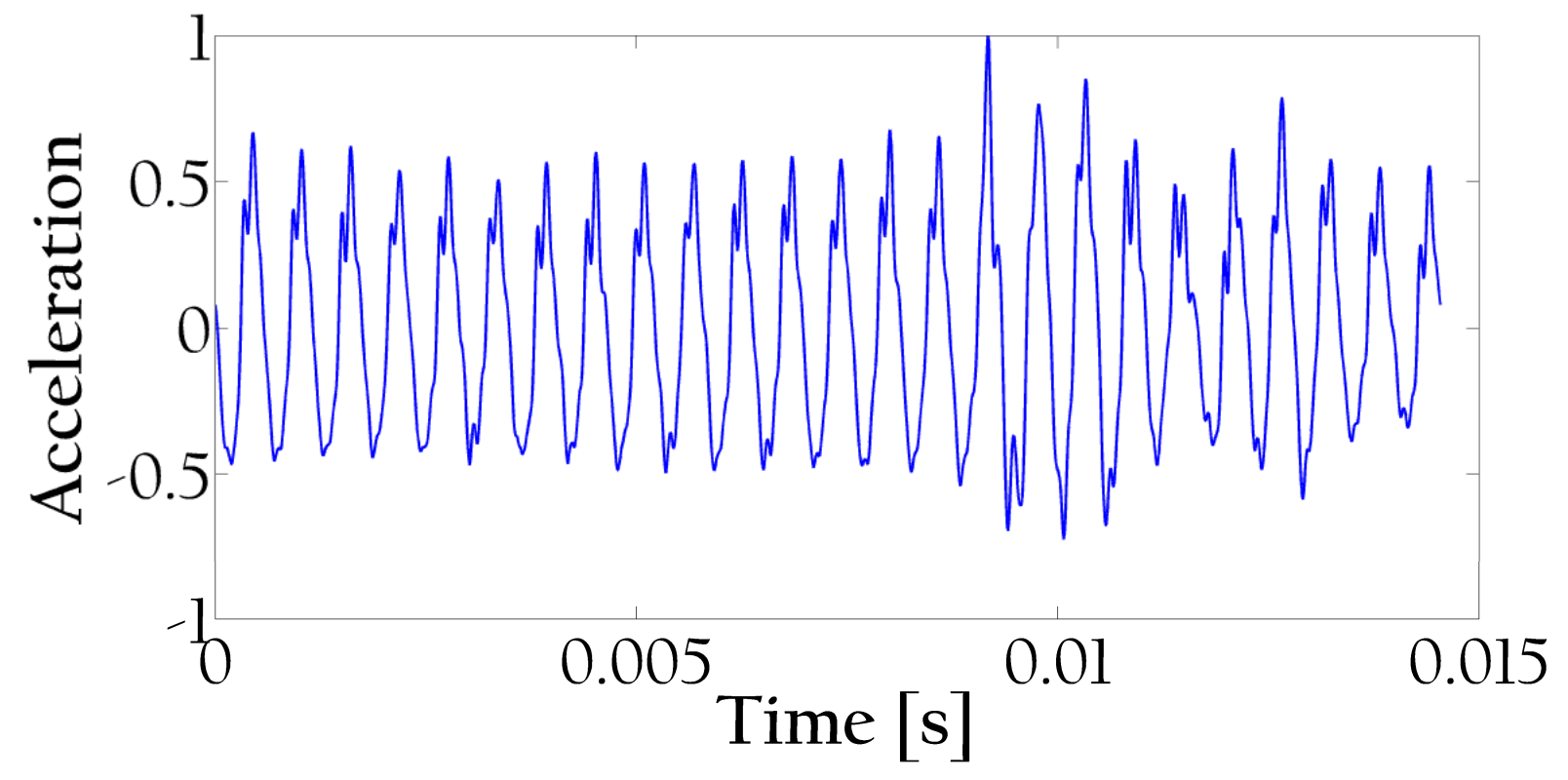

Figure 9. Normalised vibration acceleration signal derived from sample 61 


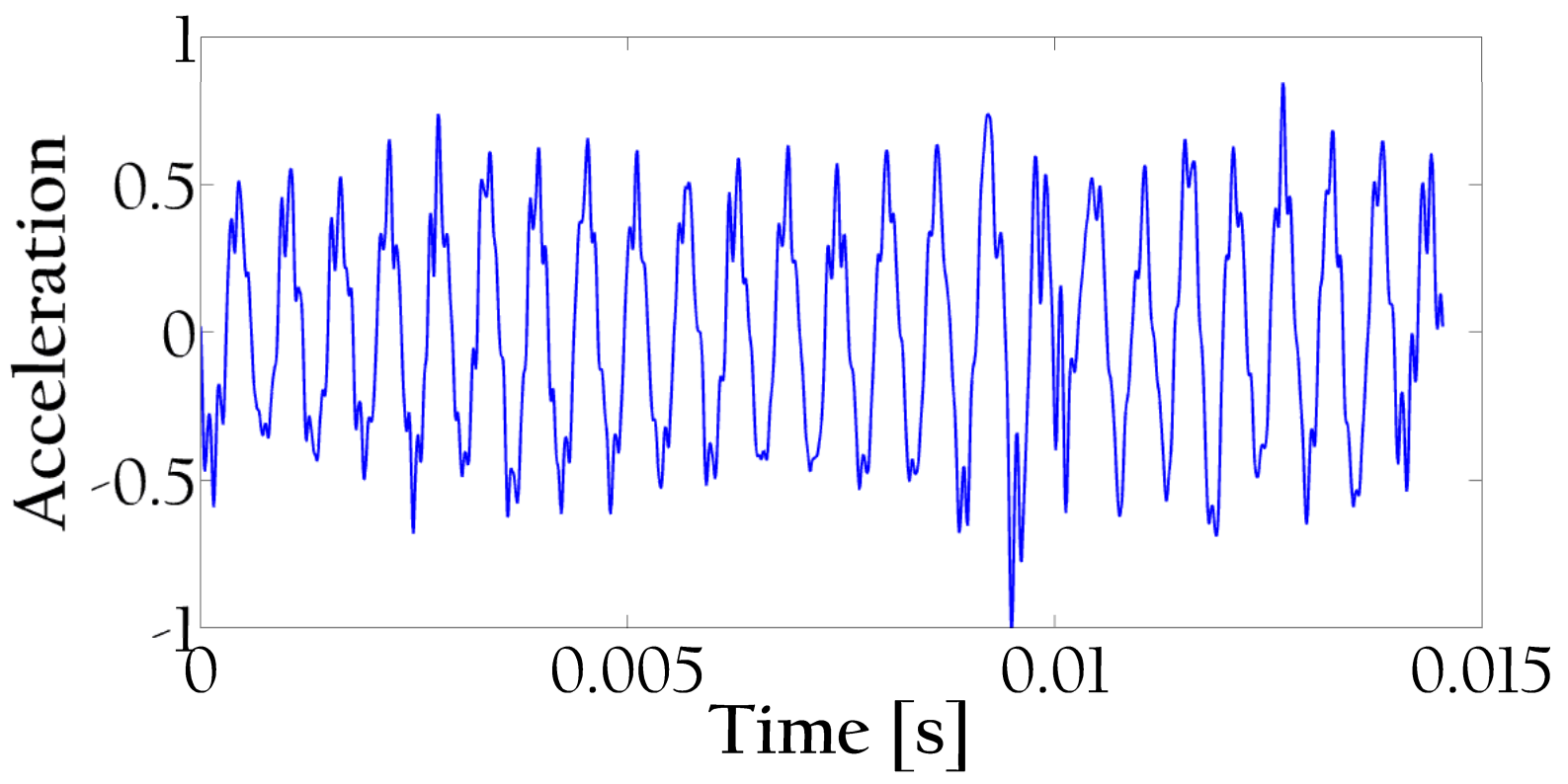

